\title{
Central Neurogenic Hyperventilation in Anti-NMDA Receptor Encephalitis
}

\author{
Atay Vural, Ethem Murat Arsava, Nese Dericioglu and Mehmet Akif Topcuoglu
}

\begin{abstract}
Central neurogenic hyperventilation $(\mathrm{CNH})$ is a rare condition that is generally associated with infiltrative tumors of the brainstem. Respiratory dysfunction, particularly central hypoventilation, is common in anti-Nmethyl D-aspartate (NMDA) receptor encephalitis. $\mathrm{CNH}$, to the best of our knowledge, has not been described previously in this disease. A 24-year-old woman was diagnosed with anti-NMDA receptor encephalitis secondary to ovarian teratoma. In addition to the typical symptoms of the disease, recurrent CNH episodes were observed during the course of the illness, which subsided with midazolam and propofol infusion. Supportive and disease-specific treatments, including oopherectomy, plasmapheresis and intravenous immunoglobulin, provided excellent recovery. These observations suggest that NMDA receptors may play a role in the pathophysiology of $\mathrm{CNH}$.
\end{abstract}

Key words: central neurogenic hyperventilation, anti-NMDA receptor encephalitis, teratoma

(Intern Med 51: 2789-2792, 2012)

(DOI: 10.2169/internalmedicine.51.8215)

\section{Introduction}

Anti-NMDA receptor encephalitis is an immune-mediated neurological disorder that usually presents with psychiatric manifestations, memory loss, disturbed consciousness, seizures, and abnormal involuntary movements including orofacial and limb dyskinesias. Autonomic instability, including tachy/bradycardia, hyper/hypothermia, hypo/hypertension, hyperhydrosis, hypersalivation, urinary incontinence and erectile dysfunction appear during the course of the illness in most patients (1). Respiratory dysfunction, usually in the form of central hypoventilation requiring mechanical support, is another well-known manifestation of this disease.

Central neurogenic hyperventilation $(\mathrm{CNH})$ is a rare condition first described by Plum and Swanson (2). The diagnostic criteria for $\mathrm{CNH}$ include hyperventilation that persists during sleep, low arterial $\mathrm{PaCO}_{2}$, high arterial $\mathrm{PaO}_{2}$, and high arterial $\mathrm{pH}$ in the absence of drug-induced, infectious, epileptic or metabolic causes. Most of the cases of CNH reported in the literature were associated with infiltrative tumors of the brainstem (3). We herein describe an unusual occurrence of $\mathrm{CNH}$ in the setting of anti-NMDA receptor encephalitis. This observation gives us new insight into the significance of NMDA receptors in respiratory physiology.

\section{Case Report}

A 24-year-old female was admitted to a local hospital with secondarily generalized seizures that gradually increased in frequency over a period of fifteen days. At the time of her admission to the local hospital, she was noted to have a decreased arousal and deficits in short-term memory. As her seizures were refractory to sodium valproate, and occurring multiple times a day, she was intubated and started on midazolam and propofol treatment. Blood, cerebrospinal fluid (CSF) and imaging studies, including brain magnetic resonance imaging (MRI), did not reveal any pathology that might explain the encephalopathy and refractory seizures. Over the following weeks the patient was kept on intravenous anesthetic agents, since the seizures recurred each time the treatment was interrupted. She was transferred to our medical center five weeks after the onset of her initial symptoms, and three weeks after her admission to the local hospital with the diagnosis of refractory status epilepticus in the setting of presumed encephalitis. 


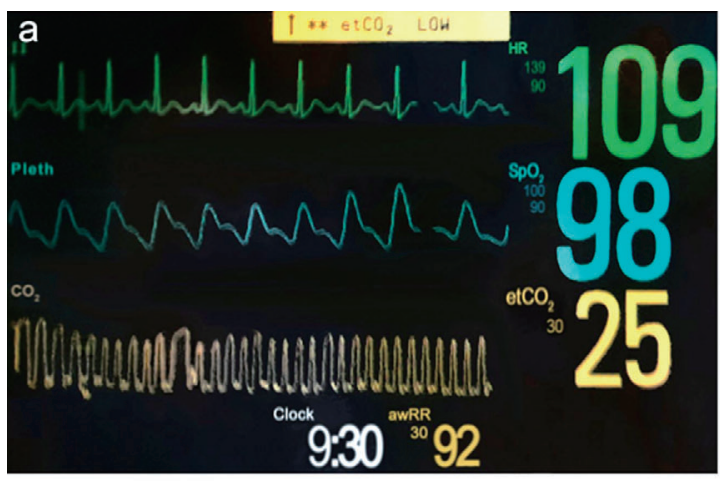

b
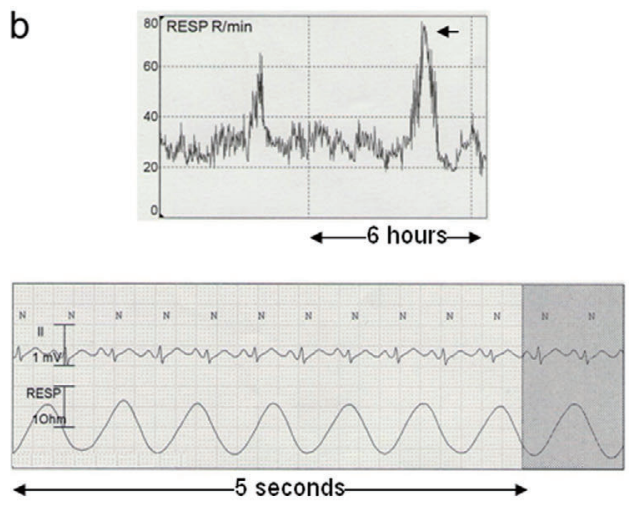

Figure. a: An epoch recorded during a $\mathrm{CNH}$ episode showed a significantly increased respiratory rate (92 breaths per minute), decreased end-tidal $\mathrm{CO} 2$ levels $(25 \mathrm{mmHg}$ ) along with normal oxygen saturation $(98 \%)$ and a slightly increased heart rate (109 beats per minute). An arterial blood gas analysis during this episode revealed the following results: $\mathrm{pH}=7.45, \mathrm{pCO} 2=29.1 \mathrm{mmHg}, \mathrm{pO} 2=86$ $\mathrm{mmHg}$ and $\mathrm{HCO3}=19,9 \mathrm{mmol} / \mathrm{L}$. b: The upper panel shows two episodes of $\mathrm{CNH}$ (each almost 1-hour in duration) during a period of 12 hours. The lower panel (a 5-second epoch recorded at the point indicated with the arrow) shows the significantly increased respiratory and heart rates, of 72 breaths per minute and 128 beats per minute.

During her evaluation upon admission to our center, she was noted to have bizarre involuntary movements of the face, tongue and extremities when the midazolam and propofol treatments were tapered off. These included lip smacking, lip pursing, chewing, licking, tongue protrusion, sucking, teeth grinding, eye blinking and choreiform movements of the extremities. She also had episodes of autonomic instability with hypersalivation, hyperhydrosis, tachycardia, mydriasis and hyperthermia. In addition, there were separate attacks of hyperventilation during which the respiratory rate increased up to 90 breaths per minute, thus jeopardizing the ventilation of the patient (Figure a, b). An arterial blood gas analysis during these episodes showed respiratory alkalosis. The duration of these $\mathrm{CNH}$ episodes was between 30-60 minutes (Figure b). They occurred up to three or four times per day, and persisted for almost three weeks, and then progressively disappeared. The $\mathrm{CNH}$ episodes could only be controlled by high dose propofol and midazolam infusion. The attacks reappeared after every cassation of the anesthetic drugs. Importantly, the respiratory rate was normal between these episodes. Of note, no epileptiform abnormalities were detected on continuous video EEG monitoring during the $\mathrm{CNH}$ episodes. There were also no accompanying autonomic instability signs or involuntary movements. CSF analysis revealed normal protein and glucose levels with no evidence for pleocytosis. Her cranial MRI on arrival was within the normal limits.

A detailed review of the patient's past medical history revealed important information that was not given by the family in prior evaluations. The patient had undergone the surgical removal of the left ovary for a mature cystic teratoma that was incidentally detected while undergoing a cesarean section for her second pregnancy. Together with the characteristic clinical picture, this information made anti-NMDA receptor encephalitis a provisional diagnosis. Pelvic ultra- sonography and MRI showed a cystic lesion suggestive of a teratoma in the right ovary. The anti-NMDA receptor antibody levels were found to be elevated both in the serum and CSF (courtesy of Dr. J. Dalmau) (4). A cystectomy was then performed and a histological examination of the surgical specimen confirmed the diagnosis of mature teratoma, which also contained hair and some cartilage tissue.

Plasmapheresis, followed by intravenous immunoglobulin treatment, was started. The midazolam and propofol treatments were gradually tapered and switched to levetiracetam. Haloperidol and clonazepam controlled the orofaciolingual and limb dyskinesias. The dyskinesias and autonomic episodes gradually improved in terms of their frequency and severity, and disappeared within several months after her surgery.

\section{Discussion}

In this report, we present the case of a patient with antiNMDA receptor encephalitis, who had $\mathrm{CNH}$ in addition to other characteristic clinical features like seizures, orofacial and limb dyskinesias, dysautonomia and encephalopathy (5). Although there have been numerous animal studies on the generation and regulation of mammalian respiratory rhythm, there have been only a few observations in humans that corroborate these findings. Almost all of the $\mathrm{CNH}$ cases reported in the literature so far were secondary to lesions involving the brainstem. Direct infiltration of the respiratory control centers in the pons and medulla or stimulation of these centers by inflammatory cytokines secreted from the neighboring tumor tissue have been suggested as the underlying pathophysiological mechanisms for $\mathrm{CNH}$ reported in these cases (3).

Recent studies have highlighted the role of NMDA receptor antibodies in glutamatergic transmission (6). The autoan- 
tibodies developed in anti-NMDA receptor encephalitis reduce the synaptic density of NMDA receptors by specifically binding to an epitope located on the extracellular domain of the NR1 subunits and perhaps, to a lesser extent, on NR2 subunits, eventually resulting in receptor cross-linking, capping and internalization (4). The decrease of synaptic NMDA receptor-mediated currents is not accompanied with a decrease in the function and density of other types of glutamate receptors, such as AMPA receptors. NMDA receptor antibodies were demonstrated to substantially enhance the excitability of the motor cortex, suggesting overactivity of glutamatergic pathways in the patients with anti-NMDA receptor encephalitis (7). This glutamatergic hyperactivity has also been shown in models of pharmacological or genetic reduction of NMDA receptors (8). In addition to this hyperglutamatergic state, which can directly explain several features specific to the early phase of this disease, such as psychotic agitation and seizures $(4,5)$, inactivation of GABAergic neurons, which actually express higher concentrations of NMDA receptors than do other neuronal subtypes, may also contribute to the clinical manifestations (4). Although this connection has yet to be demonstrated in all cases, the inactivation of the GABAergic neurons due to an antibodymediated decrease of NMDA receptors may be involved in the complexity of some abnormal movements via disinhibition of a brainstem central pattern generator (6). The same mechanism could play a role in the development of $\mathrm{CNH}$ as discussed below.

Central hypoventilation, which is common in this disease, occurs not only in comatose patients or late in the course of the disease, but also in alert patients who are in the early course of the disease. One mechanism that can lead to central hypoventilation in NMDA receptor encephalitis, taking into account the absence of proper histopathological studies, might be the presence of occult structural lesions in respiratory centers that are below the spatial resolution of MRI. Keeping this reservation in mind, both central hypoventilation and $\mathrm{CNH}$ can also be explained, at least theoretically, on the basis of a functional neurotransmitter imbalance as characterized predominantly by the presence of a hypoGABAergic tone and glutamatergic hyperactivity (4).

The hyperglutamatergic state, together with a decrease in the $\mathrm{pH}$ of the CSF, might lead to depression of the central chemoreceptor activity provided by the arcuate nucleus, the human analogue of the chemically-coded retrotrapezoid nucleus (ccRTN), in patients with NMDA receptor encephalitis $(5,9)$. The depression of the ccRTN results not only in a reduced respiratory response to central and peripheral chemoreceptors, but also in severe hypoventilation, especially during sleep. Furthermore, a sustained normal (or perhaps increased) level of activation of non-NMDA glutamatergic receptors on pump cells of the nucleus tractus solitarius, which normally relay the inhibitory slowly adapting receptors input to the ventral respiratory column (VRC) and the pons, may contribute to the development of hypoventilation (10). In these cases, arousal might increase the breath- ing rate if the respiratory rhythm generators composed of the preBötzinger complex (PBötC) and the parafacial respiratory group (pRG) are operant (11). The PBötC neurokinin1 receptor positive pre-inspiratory pacemaker cells express the type 2 vesicular glutamate transporter (VGlut2), indicating that these neurons play a role in glutamatergic transmission and might provide an excitatory stimulus to the remainder of the VRC, thus resulting in $\mathrm{CNH}$ (10). The pontine respiratory group, including the parabrachial/Kolliker fuse (PBrKF) nuclei, considered the pneumotaxic center in the older literature, contains abundant NMDA receptors and modulates breathing and ventilatory responses to hypoxia and stress $(12,13)$. The main neurons in PBrKF are postinspiratory ones which are involved in termination of the inspiratory phase. Overactivation of PBrKF results in a decrease of the inspiration period and hyperventilation, while destruction results in failure of termination of inspiration and apneustic respiration and hypoventilation (14). In the setting of a hyperglutaminergic plus hypoGABAergic state occuring in anti-NMDA receptor encephalitis, the pontine respiratory group can induce both respiratory inhibition (apnea) and facilitation (hyperventilation) in response to changes of the respiratory pattern predictated by the balance between the PBötC and Bötzinger complex (BötC). Given that the PBötC is probably partially suppressed, but BötC, which contains mainly glysinergic neurons and therefore may continue to function normally (termination of expiration on time) in this disease, transient transmitter activity changes in the PBrKF may play a role in the development of $\mathrm{CNH}$.

In summary, little is known about the pathophysiological mechanisms that lead to anti-NMDA receptor antibody encephalitis-associated respiratory abnormalities. We postulated some hypotheses that might explain these events at the level of the brainstem respiratory networks. We think that infectious, epileptic, mechanical, cardiac and psychiatric causes were appropriately excluded in the present case. The absence of other signs of autonomic excitability, such as hypersalivation, hyperhydrosis, flushing or tachycardia during the $\mathrm{CNH}$ episodes also indicate no major role of dysautonomia. At the very least, our case highlights the fact that various patterns of respiratory disturbances should be considered among the rich repertoire of psychiatric and neurological manifestations of anti-NMDA receptor antibody encephalitis. This case may be considered as a model; and further studies performed on such patients might provide new insights regarding the respiratory physiology in humans.

\section{The authors state that they have no Conflict of Interest (COI).}

\section{Acknowledgement}

The authors thank to Professor Josep Dalmau, who kindly performed the testing of the serum and CSF samples for the NMDA receptor antibodies. 


\section{References}

1. Dalmau J, Tuzun E, Wu HY, et al. Paraneoplastic anti-N-methylD-aspartate receptor encephalitis associated with ovarian teratoma. Ann Neurol 61: 25-36, 2007.

2. Plum F, Swanson AG. Central neurogenic hyperventilation in man. AMA Arch Neurol Psychiatry 81: 535-549, 1959.

3. Tarulli AW, Lim C, Bui JD, Saper CB, Alexander MP. Central neurogenic hyperventilation: a case report and discussion of pathophysiology. Arch Neurol 62: 1632-1634, 2005.

4. Dalmau J, Lancaster E, Martinez-Hernandez E, Rosenfeld MR, Balice-Gordon R. Clinical experience and laboratory investigations in patients with anti-NMDAR encephalitis. Lancet Neurol 10: 6374, 2011.

5. Dalmau J, Gleichman AJ, Hughes EG, et al. Anti-NMDA-receptor encephalitis: case series and analysis of the effects of antibodies. Lancet Neurol 7: 1091-1098, 2008.

6. Waters KA, Machaalani R. Role of NMDA receptors in development of respiratory control. Respir Physiol Neurobiol 149: 123130, 2005.

7. Manto M, Dalmau J, Didelot A, Rogemond V, Honnorat J. Afferent facilitation of corticomotor responses is increased by IgGs of patients with NMDA-receptor antibodies. J Neurol 258: 27-33,
2011.

8. Manto M, Dalmau J, Didelot A, Rogemond V, Honnorat J. In vivo effects of antibodies from patients with anti-NMDA receptor encephalitis: further evidence of synaptic glutamatergic dysfunction. Orphanet J Rare Dis 5: 31, 2010.

9. Guyenet PG, Mulkey DK. Retrotrapezoid nucleus and parafacial respiratory group. Respir Physiol Neurobiol 173: 244-255, 2010.

10. Alheid GF, McCrimmon DR. The chemical neuroanatomy of breathing. Respir Physiol Neurobiol 164: 3-11, 2008.

11. Smith JC, Abdala AP, Koizumi H, Rybak IA, Paton JF. Spatial and functional architecture of the mammalian brain stem respiratory network: a hierarchy of three oscillatory mechanisms. J Neurophysiol 98: 3370-3387, 2007.

12. Alheid GF, Milsom WK, McCrimmon DR. Pontine influences on breathing: an overview. Respir Physiol Neurobiol 143: 105-114, 2004.

13. Boon JA, Milsom WK. NMDA receptor-mediated processes in the Parabrachial/Kolliker fuse complex influence respiratory responses directly and indirectly via changes in cortical activation state. Respir Physiol Neurobiol 162: 63-72, 2008.

14. Harris MB, Milsom WK. Apneusis follows disruption of NMDAtype glutamate receptors in vagotomized ground squirrels. Respir Physiol Neurobiol 134: 191-207, 2003.

(C) 2012 The Japanese Society of Internal Medicine http://www.naika.or.jp/imonline/index.html 